\title{
Design of RLS Wiener Smoother and Filter for Colored Observation Noise in Linear Discrete-Time Stochastic Systems
}

\section{Seiichi Nakamori}

Department of Technical Education, Kagoshima University, Kagoshima, Japan.

Email: nakamori@edu.kagoshima-u.ac.jp

Received May $16^{\text {th }}, 2012$; revised June $26^{\text {th }}, 2012$; accepted July $5^{\text {th }}, 2012$

\begin{abstract}
Almost estimators are designed for the white observation noise. In the estimation problems, rather than the white observation noise, there might be actual cases where the observation noise is modeled by the colored noise process. This paper examines to design a new estimation technique of recursive least-squares (RLS) Wiener fixed-point smoother and filter for colored observation noise in linear discrete-time wide-sense stationary stochastic systems. The observation $y(k)$ is given as the sum of the signal $z(k)=H x(k)$ and the colored observation noise $v_{c}(k)$. The RLS Wiener estimators explicitly require the following information: 1$)$ the system matrix for the state vector $x(k)$; 2$)$ the observation matrix $H$; 3) the variance of the state vector $x(k)$; 4) the system matrix for the colored observation noise $v_{c}(k)$; 5) the variance of the colored observation noise; 6 ) the input noise variance in the state equation for the colored observation noise.
\end{abstract}

Keywords: Discrete-Time Stochastic System; RLS Wiener Filte; RLS Wiener Fixed-Point Smoother; Colored Observation Noise; Covariance Information

\section{Introduction}

Like the Kalman estimators, the RLS Wiener estimation problems have been researched extensively. In [1], the RLS Wiener filter and fixed-point smoother are designed in linear discrete-time systems. The estimators require the information of the system matrix, the observation vector, the variance of the state vector in the state equation for the signal and the variance of white Gaussian observation noise. By appropriate choices of observation vector and state variables, the state-space model corresponding to the autoregressive moving average (ARMA) model is introduced. Here, some elements of the system matrix consist of the AR parameters. Contrary to the Kalman estimators, the RLS Wiener estimators are advantageous in the point that the RLS Wiener estimators do not require the information of the input noise variance and the input matrix in the state equation for the state vector. The less information in the estimators might avoid the degradation in the estimation accuracy caused by the inaccurate information regarding the state-space model. Similarly, the Chandrasekhar-type RLS Wiener fixed-point smoother, filter and predictor [2], the square- root RLS Wiener fixed-point smoother and filter [3], the RLS Wiener fixed-lag smoother [4] and the RLS Wiener FIR filter [5] have been proposed in linear discrete-time stochastic systems.

Almost estimators are designed for the white observation noise. In the estimation problems, rather than the white observation noise, there might be actual cases where the observation noise is modeled by the colored noise process. The estimation problem for the observation equation with additive colored observation noise has received much attention in the research areas of detection and estimation for communication systems. In [6-9], the estimation problem is considered in linear discrete-time stochastic systems. In [6], the speech signal is estimated by subtracting the noise bias calculated during nonspeech activity. The method can be applied as a preprocessor to narrow-band voice communication systems, speech recognition systems, or speaker authentication systems. In [7], the adaptive filtering of colored observation noise based on the Kalman filter, using the neural network, is proposed. The method is applied to restore the cephalometric images of stomatology. In [8], in the case of colored observation noise, the new observation equation, 
whose observation noise is white, is obtained by subtracting the state equation, for the colored observation noise, from the observed value. Hence, the Kalman filter is applied to the new observation equation. In [9], an alternative method is proposed with regards to the traditional handling of the autoregressive colored observation noise in Kalman filter based speech enhancement algorithm. A constrained sequential EM algorithm is proposed in [10], in which Rao-Blackwellized particle filters (RBPFs) are used in the E-step and model parameters are updated in the M-step under positivity constraints for noise variance parameters.

In [11], the estimation problem of the signal for the white observation noise is considered in linear continuous systems. Also, the spectral factorization method is discussed on the system matrix, the input matrix and the observation matrix. The innovations state-space model for the colored observation model is developed.

In [12], an improved least-squares based method is proposed for a noisy autoregressive (AR) signal using observations corrupted with colored noise.

In spite of the fruitfulness as aforementioned above, in the area of the estimation problems for the colored observation noise, the studies on the RLS Wiener estimation problems in discrete-time stochastic systems might not be seen hitherto in discrete-time stochastic systems. From this viewpoint, this paper, especially, examines to design a new estimation technique of recursive leastsquares (RLS) Wiener fixed-point smoother and filter for the colored observation noise in linear discrete-time wide-sense stationary stochastic systems. The observation $y(k)$ is given as the sum of the signal

$z(k)=H x(k)$ and the colored observation noise $v_{c}(k)$. The RLS Wiener estimators explicitly require the following information: 1$)$ the system matrix for the state vector $x(k)$; 2) the observation matrix $H$; 3) the variance of the state vector $x(k) ; 4)$ the system matrix for the colored observation noise $v_{c}(k)$; 5$)$ the variance of the colored observation noise; 6 ) the input noise variance in the state equation for the colored observation noise. Also, the filtering error variance function is proposed for the current RLS Wiener filter.

A numerical simulation example, in Section 4, shows the estimation characteristics of the current fixed-point smoother and filter for the colored observation noise.

\section{Least-Squares Fixed-Point Smoothing Problem}

Let an m-dimensional observation equation be given by

$$
y(k)=z(k)+v_{c}(k), z(k)=H x(k)
$$

in linear discrete-time stochastic systems. Here, $H$ is an $m \times n$ observation matrix, $z(k)$ is a signal and $v_{c}(k)$ is a colored observation noise. It is assumed that the signal is uncorrelated with the colored observation noise as

$$
E\left[z(k) v_{c}^{T}(s)\right]=0,0 \leq k, s<\infty .
$$

Let $K_{x}(k, s)=K_{x}(k-s)$ represent the auto-covariance function of the state vector $x(k)$ in wide-sense stationary stochastic systems [13], and let $K_{x}(k, s)$ be expressed in the form of

$$
\begin{gathered}
K_{x}(k, s)= \begin{cases}A(k) B^{T}(s), & 0 \leq s \leq k, \\
B(s) A^{T}(k), & 0 \leq k \leq s,\end{cases} \\
A(k)=\Phi^{k}, B^{T}(s)=\Phi^{-s} K_{x}(s, s) .
\end{gathered}
$$

Here, $\Phi$ is the transition matrix of $x(k)$.

Let the state-space model for $x(k)$ be described as

$$
\begin{aligned}
& x(k+1)=\Phi x(k)+G w(k), \\
& E\left[w(k) w^{T}(s)\right]=Q(k) \delta_{K}(k-s),
\end{aligned}
$$

where $G$ is an $n \times l$ input matrix and $w(k)$ is white input noise with the auto-covariance function of (4).

Let $K_{c}(\cdot, \cdot)$ denote the auto-covariance function of $v_{c}(k)$. The auto-covariance function $K_{c}(k, s)$ is given by

$$
\begin{aligned}
K_{c}(k, s) & = \begin{cases}A_{c}(k) B_{c}^{T}(s), & 0 \leq s \leq k, \\
B_{c}(s) A_{c}^{T}(t), & 0 \leq k \leq s,\end{cases} \\
A_{c}(k) & =\Phi_{c}^{k}, \quad B_{c}^{T}(s)=\Phi_{c}^{-s} K_{c}(s, s) .
\end{aligned}
$$

Let the state equation for $v_{c}(k)$ be given by

$$
\begin{aligned}
& v_{c}(k+1)=\Phi_{c} v_{c}(k)+u(k), \\
& E\left[u(k) u^{T}(s)\right]=R_{u}(k) \delta_{K}(k-s),
\end{aligned}
$$

in terms of the white input noise $u(k)$ with the variance $R_{u}$. It is found that for the expressions

$$
\begin{aligned}
K_{c}(k+1, k+1) & =E\left[v_{c}(k+1) v_{c}^{T}(k+1)\right], \\
K_{c}(k, k) & =E\left[v_{c}(k) v_{c}^{T}(k)\right],
\end{aligned}
$$

in the wide-sense stationary stochastic systems, the following relationships hold.

$$
\begin{aligned}
& R_{u}(k)=K_{c}(k+1, k+1)-\Phi_{c} K_{c}(k, k) \Phi_{c}^{T}, \\
& K_{c}(k+1, k+1)=K_{c}(k, k)=K_{c}(0)
\end{aligned}
$$

Let the fixed-point smoothing estimate $\hat{x}(k, L)$ of $x(k)$ at the fixed point $k$ be expressed by

$$
\hat{x}(k, L)=\sum_{i=1}^{L} h(k, i, L) y(i)
$$

in terms of the observed values $\{y(i), 1 \leq i \leq L\}$. In (8), 
$h(k, i, L)$ is a time-varying impulse response function.

Let us consider the estimation problem, which minimizes the mean-square value (MSV)

$$
J=E\left[\|x(k)-\hat{x}(k, L)\|^{2}\right]
$$

of the fixed-point smoothing error. From an orthogonal projection lemma [13],

$$
x(k)-\sum_{i=1}^{L} h(k, i, L) y(i) \perp y(s), 1 \leq s \leq L,
$$

the impulse response function satisfies the Wiener-Hopf equation

$$
E\left[x(k) y^{T}(s)\right]=\sum_{i=1}^{L} h(k, i, L) E\left[y(i) y^{T}(s)\right] .
$$

Here “ $\perp$ ” denotes the notation of the orthogonality. Also, from (3) and (5), $E\left[y(i) y^{T}(s)\right]$ is rewritten as

$$
\begin{aligned}
& E\left[y(i) y^{T}(s)\right] \\
& =E\left[\left(H x(i)+v_{c}(i)\right)\left(H x(s)+v_{c}(s)\right)^{T}\right] \\
& =H K_{x}(i, s) H^{T}+K_{c}(i, s) .
\end{aligned}
$$

Substituting (12) into (11), we obtain

$$
K_{x}(k, s) H^{T}=\sum_{i=1}^{L} h(k, i, L)\left(H K_{x}(i, s) H^{T}+K_{c}(i, s)\right) \text {. }
$$

In terms of the expressions of

$$
K_{v u}(i, s)=E\left[v_{c}(i) u^{T}(s)\right], K_{u v}(i, s)=E\left[u(i) v_{c}^{T}(s)\right],
$$

it is shown that

$$
\begin{aligned}
& K_{c}(i, s)=E\left[v_{c}(i) v_{c}^{T}(s)\right] \\
& =E\left[\left(\Phi_{c} v_{c}(i-1)+u(i-1)\right)\left(\Phi_{c} v_{c}(s-1)+u(s-1)\right)^{T}\right] \\
& =\Phi_{c} K_{c}(i, s) \Phi_{c}^{T}+\Phi_{c} K_{v u}(i, s)+K_{u v}(i, s) \Phi_{c}^{T} \\
& \quad+R_{u}(i) \delta_{K}(i-s)
\end{aligned}
$$

is valid from the wide sense stationary properties for $K_{c}(i, s), K_{v u}(i, s)$ and $K_{u v}(i, s)$. Substitution of (14) into (13) yields

$$
\begin{aligned}
K_{x}(k, s) H^{T}= & \sum_{i=1}^{L} h(k, i, L)\left(H K_{x}(i, s) H^{T}\right. \\
& +\Phi_{c} K_{c}(i, s) \Phi_{c}^{T}+\Phi_{c} K_{v u}(i, s) \\
& \left.+K_{u v}(i, s) \Phi_{c}^{T}+R_{u}(i) \delta_{K}(i-s)\right) .
\end{aligned}
$$

Consequently, the optimal impulse response function $h(k, s, L)$ satisfies

$$
\begin{aligned}
& h(k, s, L) R_{u}(s)=K_{x}(k, s) H^{T} \\
& -\sum_{i=1}^{L} h(k, i, L)\left(H K_{x}(i, s) H^{T}+\Phi_{c} K_{c}(i, s) \Phi_{c}^{T}\right. \\
& \left.+\Phi_{c} K_{v u}(i, s)+K_{u v}(i, s) \Phi_{c}^{T}\right) .
\end{aligned}
$$

\section{RLS Wiener Estimation Algorithms}

Under the linear least-squares estimation problem of the signal $z(k)$ in Section 2, Theorem 1 shows the RLS Wiener fixed-point smoothing and filtering algorithms, which use the covariance information of the signal and observation noise.

\section{Theorem 1}

Let the auto-covariance function $K_{x}(k, s)$ of the state vector $x(k)$ be expressed by (3), let the variance of the colored observation noise $v_{c}(k)$ be $K_{c}(k, k)$ and let the variance of $u(k)$ in the state equation (6) for $v_{c}(k+1)$ be $R_{u}(k)$. Then, the RLS Wiener algorithms for the fixed-point smoothing estimate at the fixed point $k$ and the filtering estimate of the signal $z(k)$ consist of (17)-(38) in linear discrete-time stochastic systems with the wide-sense stationarities.

Fixed-point smoothing estimate of the signal $z(k)$ : $\hat{z}(k, L)$

$$
\hat{z}(k, L)=H \hat{x}(k, L)
$$

Fixed-point smoothing estimate of $x(k): \hat{x}(k, L)$

$$
\hat{x}(k, L)=\hat{x}(k, L-1)+h(k, L, L)\left(y(L)-\left(H \Phi \hat{x}(L-1, L-1)+\Phi_{c}^{2} \hat{v}_{2}(L-1, L-1)+\Phi_{c} \hat{v}_{3}(L-1, L-1)\right)\right)
$$

Smoother gain: $h(k, L, L)$

$$
\begin{aligned}
h(k, L, L)= & {\left[K_{x}(k, k)\left(\Phi^{T}\right)^{L-k} H^{T}-q_{1}(k, L-1) \Phi^{T} H^{T}-q_{2}(k, L-1)\left(\Phi_{c}^{T}\right)^{2}-q_{3}(k, L-1) \Phi_{c}^{T}\right] } \\
& \times\left[R_{u}(L)+\left(H K_{x}(L, L)-\left(H \Phi S_{11}(L-1) \Phi^{T}+\left(\Phi_{c}\right)^{2} S_{21}(L-1) \Phi^{T}+\Phi_{c} S_{31}(L-1) \Phi^{T}\right)\right) H^{T}\right. \\
& +\left(\Phi_{c} K_{c}(L, L)-\left(H \Phi S_{12}(L-1) \Phi_{c}^{T}+\left(\Phi_{c}\right)^{2} S_{22}(L-1) \Phi_{c}^{T}+\Phi_{c} S_{32}(L-1) \Phi_{c}^{T}\right)\right) \Phi_{c}^{T} \\
& \left.+\left(R_{u}(L)-\left(H \Phi S_{13}(L-1) \Phi_{c}^{T}+\left(\Phi_{c}\right)^{2} S_{23}(L-1) \Phi_{c}^{T}+\Phi_{c} S_{33}(L-1) \Phi_{c}^{T}\right)\right)\right]^{-1}
\end{aligned}
$$


$q_{1}(k, L)=q_{1}(k, L-1) \Phi^{T}+h(k, L, L)\left(H K_{x}(L, L)-\left(H \Phi S_{11}(L-1) \Phi^{T}+\left(\Phi_{c}\right)^{2} S_{21}(L-1) \Phi^{T}+\Phi_{c} S_{31}(L-1) \Phi^{T}\right)\right)$,
$q_{1}(k, k)=S_{11}(k)$

$q_{2}(k, L)=q_{2}(k, L-1) \Phi_{c}^{T}+h(k, L, L)\left(\Phi_{c} K_{c}(L, L)-\left(H \Phi S_{12}(L-1) \Phi_{c}^{T}+\left(\Phi_{c}\right)^{2} S_{22}(L-1) \Phi_{c}^{T}+\Phi_{c} S_{32}(L-1) \Phi^{T}\right)\right)$,

$q_{2}(k, k)=S_{12}(k)$

$q_{3}(k, L)=q_{3}(k, L-1) \Phi_{c}^{T}+h(k, L, L)\left(R_{u}(L)-\left(H \Phi S_{13}(L-1) \Phi_{c}^{T}+\left(\Phi_{c}\right)^{2} S_{23}(L-1) \Phi_{c}^{T}+\Phi_{c} S_{33}(L-1) \Phi_{c}^{T}\right)\right)$,

$q_{3}(k, k)=S_{13}(k)$

Filtering estimate of the signal $z(L): \hat{z}(L, L)$

$$
\hat{z}(L, L)=H \hat{x}(L, L)
$$

Filtering estimate of $x(L): \hat{x}(L, L)$

$$
\begin{aligned}
& \hat{x}(L, L)=\Phi \hat{x}(L-1, L-1)+G_{1}(L)\left(y(L)-\left(H \Phi \hat{x}(L-1, L-1)+\left(\Phi_{c}\right)^{2} \hat{v}_{2}(L-1, L-1)+\Phi_{c} \hat{v}_{3}(L-1, L-1)\right)\right), \\
& \hat{x}(0,0)=0
\end{aligned}
$$

Filtering estimate of $v_{c}(L): \Phi_{c} \hat{v}_{2}(L, L)+\hat{v}_{3}(L, L)$

$$
\begin{aligned}
& \hat{v}_{2}(L, L)=\Phi_{c} \hat{v}_{2}(L-1, L-1)+G_{2}(L)\left(y(L)-\left(H \Phi \hat{x}(L-1, L-1)+\left(\Phi_{c}\right)^{2} \hat{v}_{2}(L-1, L-1)+\Phi_{c} \hat{v}_{3}(L-1, L-1)\right)\right), \\
& \hat{v}_{2}(0,0)=0 \\
& \hat{v}_{3}(L, L)=\Phi_{c} \hat{v}_{3}(L-1, L-1)+G_{3}(L)\left(y(L)-\left(H \Phi \hat{x}(L-1, L-1)+\left(\Phi_{c}\right)^{2} \hat{v}_{2}(L-1, L-1)+\Phi_{c} \hat{v}_{3}(L-1, L-1)\right)\right), \\
& \hat{v}_{3}(0,0)=0
\end{aligned}
$$

Filtering variance function of $\hat{x}(L, L): S_{11}(L) S_{11}(L)=E\left[\hat{x}(L, L) \hat{x}^{T}(L, L)\right]$

$S_{11}(L)=\Phi S_{11}(L-1) \Phi^{T}+G_{1}(L)\left(H K_{x}(L, L)-\left(H \Phi S_{11}(L-1) \Phi^{T}+\left(\Phi_{c}\right)^{2} S_{21}(L-1) \Phi^{T}+\Phi_{c} S_{31}(L-1) \Phi^{T}\right)\right)$, $S_{11}(0)=0$

Cross-variance function of $\hat{x}(L, L)$ with $\hat{v}_{2}(L, L): S_{12}(L)=E\left[\hat{x}(L, L) \hat{v}_{2}^{T}(L, L)\right]$

$S_{12}(L)=\Phi S_{12}(L-1) \Phi_{c}^{T}+G_{1}(L)\left(\Phi_{c} K_{c}(L, L)-\left(H \Phi S_{12}(L-1) \Phi_{c}^{T}+\left(\Phi_{c}\right)^{2} S_{22}(L-1) \Phi_{c}^{T}+\Phi_{c} S_{32}(L-1) \Phi_{c}^{T}\right)\right)$, $S_{12}(0)=0$

Cross-variance function of $\hat{x}(L, L)$ with $\hat{v}_{3}(L, L): S_{13}(L)=E\left[\hat{x}(L, L) \hat{v}_{3}^{T}(L, L)\right]$

$$
\begin{aligned}
& S_{13}(L)=\Phi S_{13}(L-1) \Phi_{c}^{T}+G_{1}(L)\left(R_{u}(L)-\left(H \Phi S_{13}(L-1) \Phi_{c}^{T}+\left(\Phi_{c}\right)^{2} S_{23}(L-1) \Phi_{c}^{T}+\Phi_{c} S_{33}(L-1) \Phi_{c}^{T}\right)\right), \\
& S_{13}(0)=0 \\
& S_{21}(L)=\Phi_{c} S_{21}(L-1) \Phi^{T}+G_{2}(L)\left(H K_{x}(L, L)-\left(H \Phi S_{11}(L-1) \Phi^{T}+\left(\Phi_{c}\right)^{2} S_{21}(L-1) \Phi^{T}+\Phi_{c} S_{31}(L-1) \Phi^{T}\right)\right), \\
& S_{21}(0)=0, S_{21}(L)=S_{12}^{T}(L)
\end{aligned}
$$

Filtering variance function of $\hat{v}_{2}(L, L): S_{22}(L)$

$$
\begin{aligned}
& S_{22}(L)=\Phi_{c} S_{22}(L-1) \Phi_{c}^{T}+G_{2}(L)\left(\Phi_{c} K_{c}(L, L)-\left(H \Phi S_{12}(L-1) \Phi_{c}^{T}+\left(\Phi_{c}\right)^{2} S_{22}(L-1) \Phi_{c}^{T}+\Phi_{c} S_{32}(L-1) \Phi_{c}^{T}\right)\right), \\
& S_{22}(0)=0
\end{aligned}
$$

Cross-variance function of $\hat{v}_{2}(L, L)$ with $\hat{v}_{3}(L, L): S_{23}(L)=E\left[\hat{v}_{2}(L, L) \hat{v}_{3}^{T}(L, L)\right]$ 


$$
\begin{aligned}
& S_{23}(L)=\Phi_{c} S_{23}(L-1) \Phi_{c}^{T}+G_{2}(L)\left(R_{u}(L)-\left(H \Phi S_{13}(L-1) \Phi_{c}^{T}+\left(\Phi_{c}\right)^{2} S_{23}(L-1) \Phi_{c}^{T}+\Phi_{c} S_{33}(L-1) \Phi_{c}^{T}\right)\right) \\
& S_{23}(0)=0 \\
& S_{31}(L)=\Phi_{c} S_{31}(L-1) \Phi^{T}+G_{3}(L)\left(H K_{x}(L, L)-\left(H \Phi S_{11}(L-1) \Phi^{T}+\left(\Phi_{c}\right)^{2} S_{21}(L-1) \Phi^{T}+\Phi_{c} S_{31}(L-1) \Phi^{T}\right)\right) \\
& S_{31}(0)=0, S_{31}(L)=S_{13}^{T}(L) \\
& S_{32}(L)=\Phi_{c} S_{32}(L-1) \Phi_{c}^{T}+G_{3}(L)\left(\Phi_{c} K_{c}(L, L)-\left(H \Phi S_{12}(L-1) \Phi_{c}^{T}+\left(\Phi_{c}\right)^{2} S_{22}(L-1) \Phi_{c}^{T}+\Phi_{c} S_{32}(L-1) \Phi_{c}^{T}\right)\right) \\
& S_{32}(0)=0, S_{32}(L)=S_{23}^{T}(L)
\end{aligned}
$$

Filtering variance function of $\hat{v}_{3}(L, L): S_{33}(L)=E\left[\hat{v}_{3}(L, L) \hat{v}_{3}^{T}(L, L)\right]$

$$
\begin{aligned}
& S_{33}(L)=\Phi_{c} S_{33}(L-1) \Phi_{c}^{T}+G_{3}(L)\left(R_{u}(L)-\left(H \Phi S_{13}(L-1) \Phi_{c}^{T}+\left(\Phi_{c}\right)^{2} S_{23}(L-1) \Phi_{c}^{T}+\Phi_{c} S_{33}(L-1) \Phi_{c}^{T}\right)\right), \\
& S_{33}(0)=0
\end{aligned}
$$

Filter gain for $\hat{x}(L, L): G_{1}(L)$

$$
\begin{aligned}
G_{1}(L)= & {\left[K_{x}(L, L) H^{T}-\Phi S_{11}(L-1) \Phi^{T} H^{T}-\Phi S_{12}(L-1)\left(\Phi_{c}^{T}\right)^{2}-\Phi S_{13}(L-1) \Phi_{c}^{T}\right] } \\
& \times\left[R_{u}(L)+\left(H K_{x}(L, L)-\left(H \Phi S_{11}(L-1) \Phi^{T}+\left(\Phi_{c}\right)^{2} S_{21}(L-1) \Phi^{T}+\Phi_{c} S_{31}(L-1) \Phi^{T}\right)\right) H^{T}\right. \\
& +\left(\Phi_{c} K_{c}(L, L)-\left(H \Phi S_{12}(L-1) \Phi_{c}^{T}+\left(\Phi_{c}\right)^{2} S_{22}(L-1) \Phi_{c}^{T}+\Phi_{c} S_{32}(L-1) \Phi_{c}^{T}\right)\right) \Phi_{c}^{T} \\
& \left.+\left(R_{u}(L)-\left(H \Phi S_{13}(L-1) \Phi_{c}^{T}+\left(\Phi_{c}\right)^{2} S_{23}(L-1) \Phi_{c}^{T}+\Phi_{c} S_{33}(L-1) \Phi_{c}^{T}\right)\right)\right]^{-1}
\end{aligned}
$$

Filter gain for $\hat{v}_{2}(L, L): G_{2}(L)$

$$
\begin{aligned}
G_{2}(L)= & {\left[K_{c}(L, L) \Phi_{c}^{T}-\Phi_{c} S_{21}(L-1) \Phi^{T} H^{T}-\Phi_{c} S_{22}(L-1)\left(\Phi_{c}^{T}\right)^{2}-\Phi_{c} S_{23}(L-1) \Phi_{c}^{T}\right] } \\
& \times\left[R_{u}(L)+\left(H K_{x}(L, L)-\left(H \Phi S_{11}(L-1) \Phi^{T}+\left(\Phi_{c}\right)^{2} S_{21}(L-1) \Phi^{T}+\Phi_{c} S_{31}(L-1) \Phi^{T}\right)\right) H^{T}\right. \\
& +\left(\Phi_{c} K_{c}(L, L)-\left(H \Phi S_{12}(L-1) \Phi_{c}^{T}+\left(\Phi_{c}\right)^{2} S_{22}(L-1) \Phi_{c}^{T}+\Phi_{c} S_{32}(L-1) \Phi_{c}^{T}\right)\right) \Phi_{c}^{T} \\
& \left.+\left(R_{u}(L)-\left(H \Phi S_{13}(L-1) \Phi_{c}^{T}+\left(\Phi_{c}\right)^{2} S_{23}(L-1) \Phi_{c}^{T}+\Phi_{c} S_{33}(L-1) \Phi_{c}^{T}\right)\right)\right]^{-1}
\end{aligned}
$$

Filter gain for $\hat{v}_{3}(L, L): G_{3}(L)$

$$
\begin{aligned}
G_{3}(L)= & {\left[R_{u}(L)-\Phi_{c} S_{31}(L-1) \Phi^{T} H^{T}-\Phi_{c} S_{32}(L-1)\left(\Phi_{c}^{T}\right)^{2}-\Phi_{c} S_{33}(L-1) \Phi_{c}^{T}\right] } \\
& \times\left[R_{u}(L)+\left(H K_{x}(L, L)-\left(H \Phi S_{11}(L-1) \Phi^{T}+\left(\Phi_{c}\right)^{2} S_{21}(L-1) \Phi^{T}+\Phi_{c} S_{31}(L-1) \Phi^{T}\right)\right) H^{T}\right. \\
& +\left(\Phi_{c} K_{c}(L, L)-\left(H \Phi S_{12}(L-1) \Phi_{c}^{T}+\left(\Phi_{c}\right)^{2} S_{22}(L-1) \Phi_{c}^{T}+\Phi_{c} S_{32}(L-1) \Phi_{c}^{T}\right)\right) \Phi_{c}^{T} \\
& \left.+\left(R_{u}(L)-\left(H \Phi S_{13}(L-1) \Phi_{c}^{T}+\left(\Phi_{c}\right)^{2} S_{23}(L-1) \Phi_{c}^{T}+\Phi_{c} S_{33}(L-1) \Phi_{c}^{T}\right)\right)\right]^{-1}
\end{aligned}
$$

Proof of Theorem 1 is deferred to the Appendix.

Figure 1 illustrates the flowchart for the filtering and fixed-point smoothing algorithms of Theorem 1. The calculating steps are divided into two parts corresponding to the filter and the fixed-point smoother.

From Theorem 1, it is found that the filtering error variance function $\tilde{P}_{z}(L)$ of the signal $z(L)$ is given by

$$
\tilde{P}_{z}(L)=K_{z}(L, L)-H \Phi S_{11}(L-1) \Phi^{T} H^{T},
$$

where $K_{z}(L, L)$ represents the variance function of $z(L)$. 


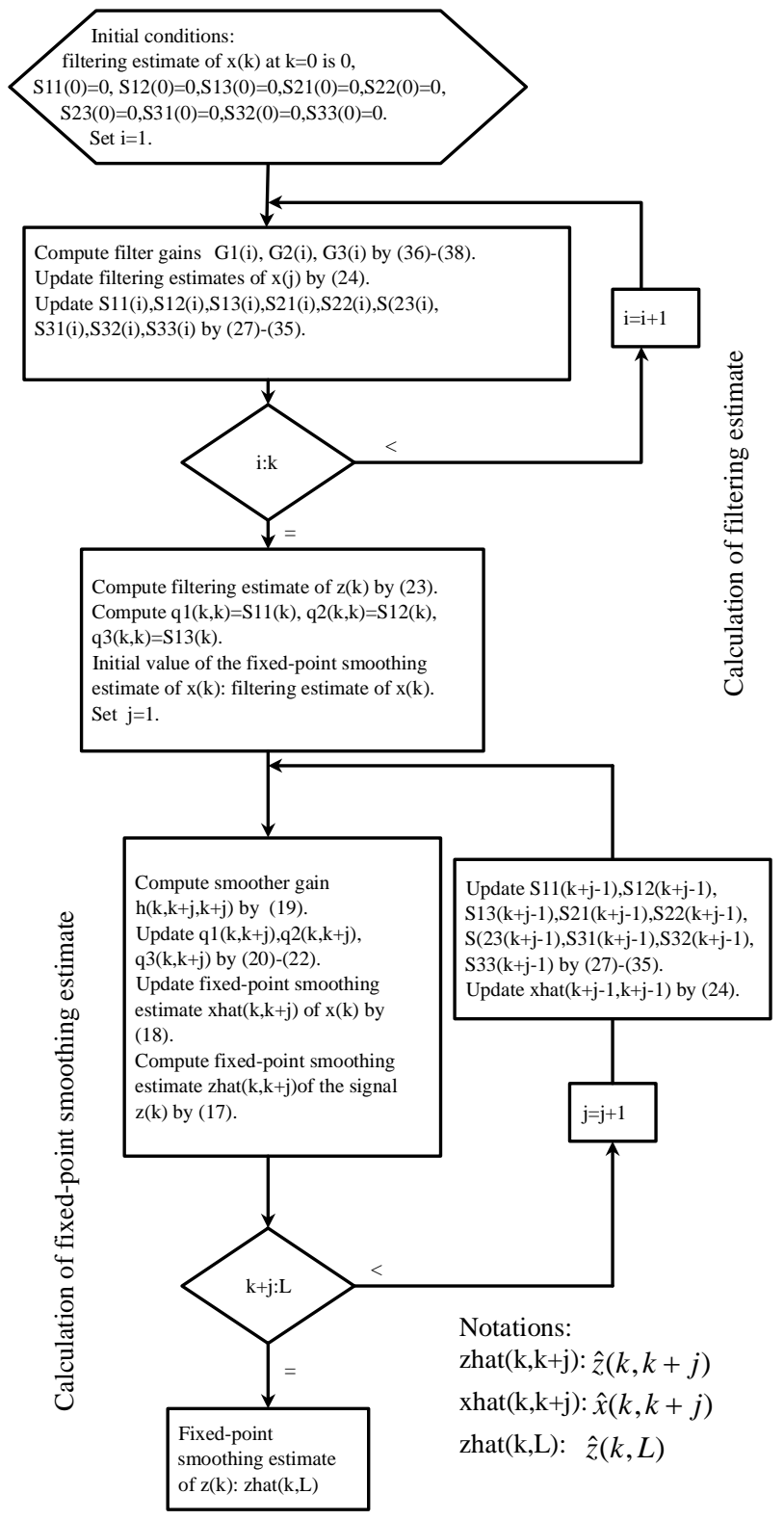

Figure 1. Flowchart for the filtering and fixed-point smoothing algorithm of Theorem 1.

\section{A Numerical Simulation Example}

In this section, to show the estimation characteristics of the proposed RLS Wiener fixed-point smoother and filter, a numerical example is presented.

Let a scalar observation equation be given by

$$
\begin{gathered}
y(k)=z(k)+v_{c}(k), \\
z(k)=H x(k) .
\end{gathered}
$$

Here, $v_{c}(k)$ is zero-mean colored observation noise. Let the signal $z(k)$ be generated by the second-order AR model.

$$
\begin{gathered}
z(k+1)=-a_{1} z(k)-a_{2} z(k+1)+w(k), \\
E[w(k) w(s)]=\sigma^{2} \delta_{K}(k-s), \\
a_{1}=-0.1, a_{2}=-0.8, \sigma=0.5 .
\end{gathered}
$$

Hence, the state-space model for $z(k)$ is given by

$$
\begin{gathered}
z(k)=H x(k)=x_{1}(k), H=\left[\begin{array}{ll}
1 & 0
\end{array}\right], x(k)=\left[\begin{array}{l}
x_{1}(k) \\
x_{2}(k)
\end{array}\right], \\
{\left[\begin{array}{l}
x_{1}(k+1) \\
x_{2}(k+1)
\end{array}\right]=\left[\begin{array}{cc}
0 & 1 \\
-a_{2} & -a_{1}
\end{array}\right]\left[\begin{array}{l}
x_{1}(k) \\
x_{2}(k)
\end{array}\right]+\left[\begin{array}{l}
0 \\
1
\end{array}\right] w(k) .}
\end{gathered}
$$

The auto-covariance function of the signal $z(k)$ is given by [11]

$$
\begin{aligned}
& K(0)=\sigma^{2} \text {, } \\
& K(m)=\sigma^{2}\left\{\alpha_{1}\left(\alpha_{2}^{2}-1\right) \alpha_{1}^{m} /\left[\left(\alpha_{2}-\alpha_{1}\right)\left(\alpha_{2} \alpha_{1}+1\right)\right]\right. \\
& \left.-\alpha_{2}\left(\alpha_{1}^{2}-1\right) \alpha_{2}^{m} /\left[\left(\alpha_{2}-\alpha_{1}\right)\left(\alpha_{1} \alpha_{2}+1\right)\right]\right\}, \\
& 0<m, \alpha_{1}, \alpha_{2}=\left(-a_{1} \pm \sqrt{a_{1}^{2}-4 a_{2}}\right) / 2 \text {. }
\end{aligned}
$$

From (43) and (44), it is seen that

$$
\begin{gathered}
K_{x}(k, k)=\left[\begin{array}{ll}
K(0) & K(1) \\
K(1) & K(0)
\end{array}\right], \Phi=\left[\begin{array}{cc}
0 & 1 \\
-a_{2} & -a_{1}
\end{array}\right], \\
K(0)=0.25, K(1)=0.125 .
\end{gathered}
$$

Let the state equation for $v_{c}(k)$ be given by

$$
\begin{gathered}
v_{c}(k+1)=\Phi_{c} v_{c}(k)+u(k), \\
E\left[u(k) u^{T}(s)\right]=R_{u}(k) \delta_{K}(k-s), \\
\Phi_{c}=0.91 .
\end{gathered}
$$

$u(k)$ is white Gaussin input noise in the state equation for the colored observation noise process. The autovariance function of the colored observation noise $v_{c}(\cdot)$ satisfies the relationships

$$
K_{c}(k+1, k+1)=K_{c}(k, k)=K_{c}(0) \text { and } K_{c}(0)=\frac{R_{u}}{1-a^{2}}
$$

in wide-sense stationary stochastic systems.

Substituting $H, \Phi, K_{x}(L, L)=K_{x}(0), \Phi_{c}$, $K_{c}(L, L)=K_{c}(0)$ and $R_{u}$ into the RLS Wiener estimation algorithms of Theorem 1 , the filtering and fixedpoint smoothing estimates are calculated recursively.

Figure 2 illustrates the colored observation noise process $v_{c}(k)$ vs. $k, 1 \leq k \leq 250$, for the variance $R_{u}=0.01$ in (45). Figure 3 illustrates the fixed-point smoothing estimate $\hat{z}(k, k+5)$ vs. $k, 1 \leq k \leq 250$, for the variance $R_{u}=0.01$. Figure 4 illustrates the MSVs of the filtering errors $z(k)-\hat{z}(k, k)$ and the fixed-point smoothing errors and $R_{u}=0.0225\left(K_{c}(0)=0.1309\right)$. As $R_{u}$ becomes large, the variance $K_{c}(0)$ of the colored observation noise becomes large. For $\operatorname{Lag}=0$, the MSV 


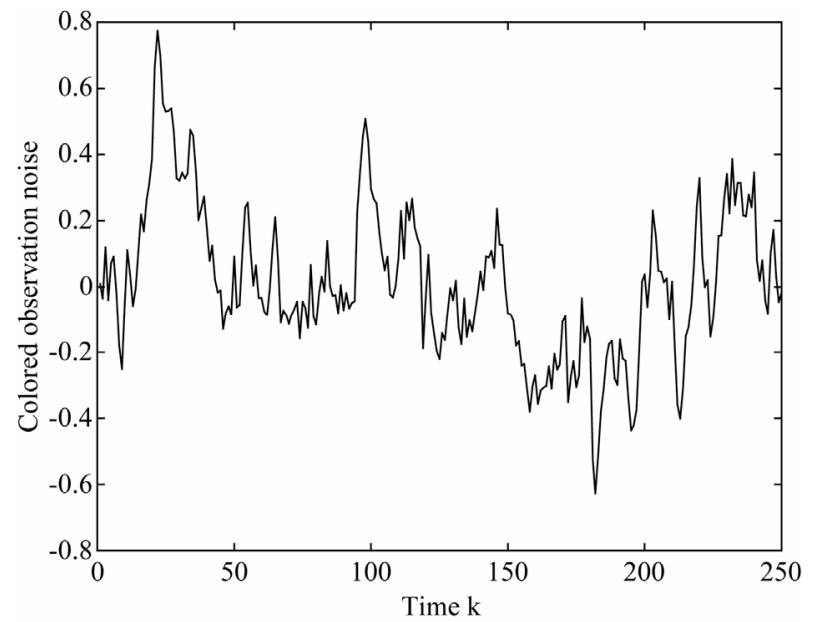

Figure 2. Colored observation noise process $v_{c}(k)$ vs. $k, 1 \leq k$ $\leq 250$, for the variance $R_{u}=0.01$ in (41).

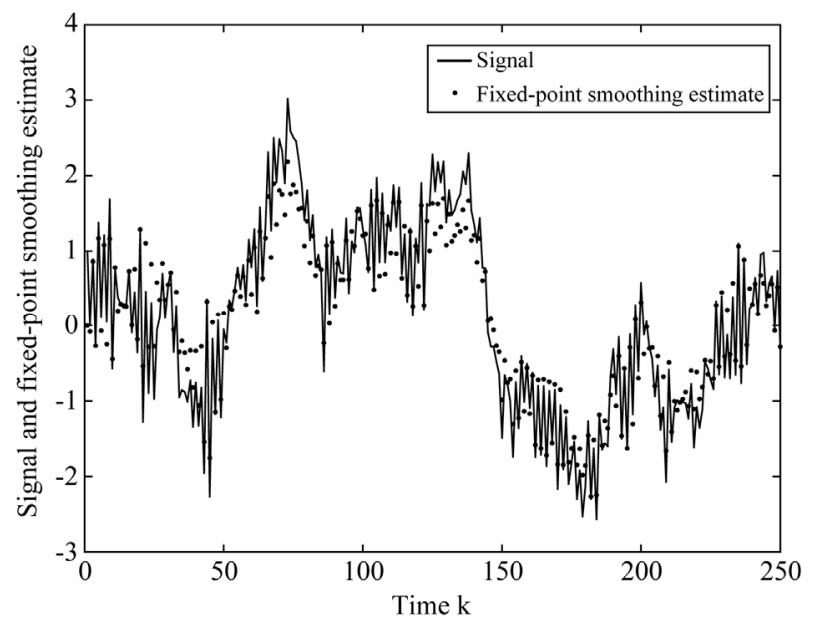

Figure 3. Fixed-point smoothing estimate $\hat{z}(k, k+5)$ vs. $k$, $1 \leq k \leq 250$, for the variance $R_{u}=0.01$.

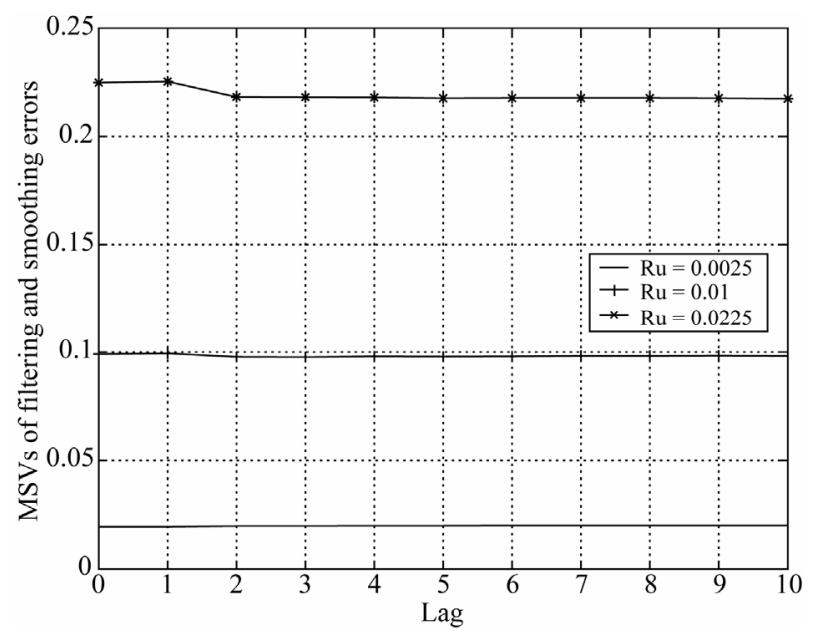

Figure 4. MSVs of the filtering errors $z(k)-\hat{z}(k, k)$ and the fixed-point smoothing errors $z(k)-\hat{z}(k, k+L a g)$ vs. Lag. of the filtering errors $z(k)-\hat{z}(k, k)$ is shown. As Lag increases, $1 \leq \operatorname{Lag} \leq 3$, the MSVs gradually decrease for $R_{u}=0.01$ and $R_{u}=0.0225$. Hence, the estimation accuracy of the fixed-point smoother is superior to that of the filter. For the colored observation noise with the larger variances $R_{u}$ of the input noise and $K_{c}(0)$ of the colored observation noise, the MSVs of the filtering errors and the fixed-point smoothing errors increase and the estimation accuracy is degraded. Here, the MSVs of the fixed-point smoothing and filtering errors are evaluated by

$$
\begin{gathered}
\sum_{k=1}^{2000}(z(k)-\hat{z}(k, k+\operatorname{Lag}))^{2} / 2000,1 \leq \operatorname{Lag} \leq 10, \text { and } \\
\sum_{k=1}^{2000}(z(k)-\hat{z}(k, k))^{2} / 2000 .
\end{gathered}
$$

\section{Conclusions}

In this paper, the RLS Wiener fixed-point smoother and filter have been designed for the colored observation noise.

A numerical simulation example shows that the proposed estimation algorithms for the RLS Wiener fixedpoint smoothing and filtering estimates, in the case of the colored observation noise, is feasible. From Figure 4, as Lag increases, $1 \leq \operatorname{Lag} \leq 3$, the MSVs gradually decrease for $R_{u}=0.01$ and $R_{u}=0.0225$. Hence, the estimation accuracy of the fixed-point smoother is superior to that of the filter.

The RLS Wiener estimators do not use the information of the variance $Q(k)$, for the input noise $w(k)$, and the input matrix $G$ in the state Equation (4), in comparison with the Kalman estimation technique [8]. In the RLS Wiener estimators, it is not necessary to take account of the degraded estimation accuracy caused by the modeling errors for $Q(k)$ and $G$.

\section{REFERENCES}

[1] S. Nakamori, "Recursive Estimation Technique of Signal from Output Measurement Data in Linear Discrete-Time Systems," IEICE Transactions on Fundamentals of Electronics, Communications and Computer Sciences, Vol. E78-A, No. 5, 1995, pp. 600-607.

[2] S. Nakamori, "Chandrasekhar-Type Recursive Wiener Estimation Technique in Linear Discrete-Time Systems," Applied Mathematics and Computation, Vol. 188, No. 2, 2007, pp. 1656-1665. doi:10.1016/j.amc.2006.11.021

[3] S. Nakamori, "Square-Root Algorithms of RLS Wiener Filter and Fixed-Point Smoother in Linear Discrete Stochastic Systems," Applied Mathematics and Computation, Vol. 203, No. 1, 2008, pp. 186-193. doi:10.1016/j.amc.2008.04.026

[4] S. Nakamori, A. Hermoso-Carazo and J. Linares-P'erez, 
"Design of RLS Wiener Fixed-Lag Smoother Using Covariance Information in Linear Discrete Stochastic Systems,” Applied Mathematical Modelling, Vol. 32, No. 7, 2008, pp. 1338-1349. doi:10.1016/j.apm.2007.04.008

[5] S. Nakamori, "Design of RLS Wiener FIR Filter Using Covariance Information in Linear Discrete-Time Stochastic Systems,” Digital Signal Processing, Vol. 20, No. 5, 2010, pp. 1310-1329. doi:10.1016/j.dsp.2010.01.001

[6] S. Boll, "Suppression of Acoustic Noise in Speech Using Spectral Subtraction," IEEE Transactions on Acoustics, Speech and Signal Processing, Vol. 27, No. 2, 1979, pp. 113-120. doi:10.1109/TASSP.1979.1163209

[7] S. S. Xiong and Z. Y. Zhou, "Neural Filtering of Colored Noise Based on Kalman Filter Structure," IEEE Transactions on Instrumentation and Measurement, Vol. 52, No. 3, 2003, pp. 742-747. doi:10.1109/TIM.2003.814669

[8] D. Simon, "Optimal State Estimation: Kalman, $\mathrm{H} \infty$, and Nonlinear Approaches,” Wiley, New York, 2006. doi:10.1002/0470045345

[9] F. Must'iere, M. Boli'c and M. Bouchad, "Improved Col- ored Noise Handling in Kalman Filter-Based Speech Enhancement Algorithms," Canadian Conference on Electrical Computer Engineering, Niagara Falls, 4-7 May 2008, pp. 497-500.

[10] S. Park and S. Choi, "A Constrained Sequential EM Algorithm for Speech Enhancement,” Neural Networks, Vol. 21, No. 9, 2008, pp. 1401-1409. doi:10.1016/j.neunet.2008.03.001

[11] S. Nakamori, "Estimation of Signal and Parameters Using Covariance Information in Linear Continuous Systems," Mathematical and Computer Modeling, Vol. 16, No. 10, 1992, pp. 3-15. doi:10.1016/0895-7177(92)90056-Q

[12] A. Mahmoudi and M. Karimi, "Parameter Estimation of Autoregressive Signals from Observations Corrupted with Colored Noise," Signal Processing, Vol. 90, No. 1, 2010, pp. 157-164. doi:10.1016/j.sigpro.2009.06.005

[13] A. P. Sage and J. L. Melsa, "Estimation Theory with Applications to Communications and Control,” McGraw-Hill, New York, 1971. 


\section{Appendix Proof of Theorem 1}

If we subtract the equation obtained by putting $L \rightarrow L-1$ in (16) from (16), we have

$$
\begin{aligned}
& (h(k, s, L)-h(k, s, L-1)) R_{u}(s)=-h(k, L, L)\left(H K_{x}(L, s) H^{T}+\Phi_{c} K_{c}(L, s) \Phi_{v}^{T}+\Phi_{c} K_{v u}(L, s)\right) \\
& -\sum_{i=1}^{L-1}(h(k, i, L)-h(k, i, L-1))\left(H K_{x}(i, s) H^{T}+\Phi_{c} K_{c}(i, s) \Phi_{c}^{T}+\Phi_{c} K_{v u}(i, s)+K_{u v}(i, s) \Phi_{c}^{T}\right),
\end{aligned}
$$

for $K_{u v}(L, s)=0$.

From (6) and $K_{v u}(s, s)=0$, it is shown that $K_{v u}(L, s)$ satisfies

$$
K_{v u}(L, s)=\Phi_{c}^{L-s} K_{v u}(s, s)+\Phi_{c}^{L-s-1} R_{u}(s)=\Phi_{c}^{L-s-1} R_{u}(s) .
$$

Let us introduce the following auxiliary functions $J_{1}(s, L-1), J_{2}(s, L-1)$ and $J_{3}(s, L-1)$ as follows.

$J_{1}(s, L-1) R_{u}(s)=\Phi^{-s} K_{x}(s, s) H^{T}-\sum_{i=1}^{L-1} J_{1}(i, L-1)\left(H K_{x}(i, s) H^{T}+\Phi_{c} K_{c}(i, s) \Phi_{c}^{T}+\Phi_{c} K_{v u}(i, s)+K_{u v}(i, s) \Phi_{c}^{T}\right)$,

$J_{2}(s, L-1) R_{u}(s)=\Phi_{c}^{-s} K_{c}(s, s) \Phi_{c}^{T}-\sum_{i=1}^{L-1} J_{2}(i, L-1)\left(H K_{x}(i, s) H^{T}+\Phi_{c} K_{c}(i, s) \Phi_{c}^{T}+\Phi_{c} K_{v u}(i, s)+K_{u v}(i, s) \Phi_{c}^{T}\right)$,

$J_{3}(s, L-1) R_{u}(s)=\Phi_{c}^{-s} R_{u}(s)-\sum_{i=1}^{L-1} J_{3}(i, L-1)\left(H K_{x}(i, s) H^{T}+\Phi_{c} K_{c}(i, s) \Phi_{c}^{T}+\Phi_{c} K_{v u}(i, s)+K_{u v}(i, s) \Phi_{c}^{T}\right)$.

From (A-1), (A-3), (A-4) and (A-5), we obtain

$$
h(k, s, L)-h(k, s, L-1)=-h(k, L, L)\left(H \Phi^{L} J_{1}(s, L-1)+\Phi_{c}^{L+1} J_{2}(s, L-1)+\Phi_{c}^{L} J_{3}(s, L-1)\right) .
$$

If we subtract $J_{1}(s, L-1) R_{u}(s)$ from $J_{1}(s, L) R_{u}(s)$, we have

$$
\begin{aligned}
& \left(J_{1}(s, L)-J_{1}(s, L-1)\right) R_{u}(s)=-J_{1}(L, L)\left(H K_{x}(L, s) H^{T}+\Phi_{c} K_{c}(L, s) \Phi_{c}^{T}+\Phi_{c} K_{v u}(L, s)+K_{u v}(L, s) \Phi_{c}^{T}\right) \\
& -\sum_{i=1}^{L-1}\left(J_{1}(i, L)-J_{1}(i, L-1)\right)\left(H K_{x}(i, s) H^{T}+\Phi_{c} K_{c}(i, s) \Phi_{c}^{T}+\Phi_{c} K_{v u}(i, s)+K_{u v}(i, s) \Phi_{c}^{T}\right) .
\end{aligned}
$$

From (A-3)-(A-5) and (A-7), we obtain

$$
J_{1}(s, L)=J_{1}(s, L-1)-J_{1}(L, L)\left(H \Phi^{L} J_{1}(s, L-1)+\Phi_{c}^{L+1} J_{2}(s, L-1)+\Phi_{c}^{L} J_{3}(s, L-1)\right) .
$$

If we subtract $J_{2}(s, L-1) \bar{R}(s)$ from $J_{2}(s, L) \bar{R}(s)$, we have

$$
\begin{aligned}
& \left(J_{2}(s, L)-J_{2}(s, L-1)\right) R_{u}(s)=-J_{2}(L, L)\left(H K_{x}(L, s) H^{T}+\Phi_{c} K_{c}(L, s) \Phi_{c}^{T}+\Phi_{c} K_{v u}(L, s)+K_{u v}(L, s) \Phi_{c}^{T}\right) \\
& -\sum_{i=1}^{L-1}\left(J_{2}(i, L)-J_{2}(i, L-1)\right)\left(H K_{x}(i, s) H^{T}+\Phi_{c} K_{c}(i, s) \Phi_{c}^{T}+\Phi_{c} K_{v u}(i, s)+K_{u v}(i, s) \Phi_{c}^{T}\right) .
\end{aligned}
$$

From (A-3)-(A-5) and (A-9), we obtain

$$
J_{2}(s, L)=J_{2}(s, L-1)-J_{2}(L, L)\left(H \Phi^{L} J_{1}(s, L-1)+\Phi_{c}^{L+1} J_{2}(s, L-1)+\Phi_{c}^{L} J_{3}(s, L-1)\right) .
$$

If we subtract $J_{3}(s, L-1) R_{u}(s)$ from $J_{3}(s, L) R_{u}(s)$, we have

$$
\begin{aligned}
& \left(J_{3}(s, L)-J_{3}(s, L-1)\right) R_{u}(s)=-J_{3}(L, L)\left(H K_{x}(L, s) H^{T}+\Phi_{c} K_{c}(L, s) \Phi_{c}^{T}+\Phi_{c} K_{v u}(L, s)+K_{u v}(L, s) \Phi_{c}^{T}\right) \\
& -\sum_{i=1}^{L-1}\left(J_{3}(i, L)-J_{3}(i, L-1)\right)\left(H K_{x}(i, s) H^{T}+\Phi_{c} K_{c}(i, s) \Phi_{c}^{T}+\Phi_{c} K_{v u}(i, s)+K_{u v}(i, s) \Phi_{c}^{T}\right) .
\end{aligned}
$$

From (A-3)-(A-5) and (A-11), we obtain

$$
J_{3}(s, L)=J_{3}(s, L-1)-J_{3}(L, L)\left(H \Phi^{L} J_{1}(s, L-1)+\Phi_{c}^{L+1} J_{2}(s, L-1)+\Phi_{c}^{L} J_{3}(s, L-1)\right) .
$$

By putting $s=L-1$ in (A-3), we have 


$$
\begin{aligned}
& J_{1}(L-1, L-1) R_{u}(L-1) \\
& =\Phi^{-(L-1)} K_{x}(L-1, L-1) H^{T}-\sum_{i=1}^{L-1} J_{1}(i, L-1)\left(H K_{x}(i, L-1) H^{T}+\Phi_{c} K_{c}(i, L-1) \Phi_{c}^{T}+\Phi_{c} K_{v u}(i, L-1)+K_{u v}(i, L-1) \Phi_{c}^{T}\right) \\
& =\Phi^{-(L-1)} K_{x}(L-1, L-1) H^{T}-\sum_{i=1}^{L-1} J_{1}(i, L-1)\left(B(i) A^{T}(L-1) H^{T}+\Phi_{c} B_{c}(i) A_{c}^{T}(L-1) \Phi_{c}^{T}+B_{u v}(i)\left(\Phi_{c}^{T}\right)^{L-2} \Phi_{c}^{T}\right) .
\end{aligned}
$$

Here, from (6), the relationships $K_{v u}(i, L-1)=0, \quad 1 \leq i \leq L-1$, and $K_{u v}(i, L-1)=B_{u v}(i)\left(\Phi_{c}^{T}\right)^{L-2}$, $B_{u v}(i)=R_{u}(i)\left(\Phi_{v}^{T}\right)^{-i}$ are taken into accounts.

By introducing the functions

$$
\begin{gathered}
r_{11}(L-1)=\sum_{i=1}^{L-1} J_{1}(i, L-1) H B(i), \\
r_{12}(L-1)=\sum_{i=1}^{L-1} J_{1}(i, L-1) \Phi_{c} B_{c v}(i), \\
r_{13}(L-1)=\sum_{i=1}^{L-1} J_{1}(i, L-1) B_{u v}(i),
\end{gathered}
$$

(A-13) can be written as

$$
\begin{aligned}
J_{1}(L-1, L-1) R_{u}(L-1)= & \Phi^{-(L-1)} K_{x}(L-1, L-1) H^{T} \\
& -\left(r_{11}(L-1) A^{T}(L-1) H^{T}+r_{12}(L-1) A_{c}^{T}(L-1) \Phi_{c}^{T}+r_{12}(L-1)\left(\Phi_{c}^{T}\right)^{(L-1)}\right) .
\end{aligned}
$$

By putting $s=L-1$ in (A-4), we have

$$
\begin{aligned}
& J_{2}(L-1, L-1) R_{u}(L-1)=\Phi_{c}^{-(L-1)} K_{c}(L-1, L-1) \Phi_{c}^{T} \\
& -\sum_{i=1}^{L-1} J_{2}(i, L-1)\left(H K_{x}(i, L-1) H^{T}+\Phi_{c} K_{c}(i, L-1) \Phi_{c}^{T}+\Phi_{c} K_{v u}(i, L-1)+K_{u v}(i, L-1) \Phi_{c}^{T}\right) .
\end{aligned}
$$

By introducing the functions

$$
\begin{aligned}
& r_{21}(L-1)=\sum_{i=1}^{L-1} J_{2}(i, L-1) H B(i), \\
& r_{22}(L-1)=\sum_{i=1}^{L-1} J_{2}(i, L-1) \Phi_{c} B_{c}(i), \\
& r_{23}(L-1)=\sum_{i=1}^{L-1} J_{2}(i, L-1) B_{u v}(i),
\end{aligned}
$$

(A-18) can be written as

$$
\begin{aligned}
J_{2}(L-1, L-1) R_{u}(L-1)= & \Phi_{c}^{-(L-1)} K_{c}(L-1, L-1) \Phi_{c}^{T} \\
& -\left(r_{21}(L-1) A^{T}(L-1) H^{T}+r_{22}(L-1) A_{c}^{T}(L-1) \Phi_{c}^{T}+r_{23}(L-1)\left(\Phi_{c}^{T}\right)^{L-1}\right) .
\end{aligned}
$$

By putting $s=L-1$ in (A-5), we have

$$
\begin{aligned}
& J_{3}(L-1, L-1) R_{u}(L-1)=\Phi_{c}^{-(L-1)} R_{u}(L-1) H^{T} \\
& -\sum_{i=1}^{L-1} J_{3}(i, L-1)\left(H K_{x}(i, L-1) H^{T}+\Phi_{c} K_{c}(i, L-1) \Phi_{c}^{T}+\Phi_{c} K_{v u}(i, L-1)+K_{u v}(i, L-1) \Phi_{c}^{T}\right) .
\end{aligned}
$$

By introducing the functions 


$$
\begin{aligned}
& r_{31}(L-1)=\sum_{i=1}^{L-1} J_{3}(i, L-1) H B(i), \\
& r_{32}(L-1)=\sum_{i=1}^{L-1} J_{3}(i, L-1) \Phi_{c} B_{c}(i), \\
& r_{33}(L-1)=\sum_{i=1}^{L-1} J_{3}(i, L-1) B_{u v}(i),
\end{aligned}
$$

(A-23) can be written as

$$
\begin{aligned}
J_{3}(L-1, L-1) R(L-1)= & \Phi_{c}^{-(L-1)} R_{u}(L-1) \\
& -\left(r_{31}(L-1) A^{T}(L-1) H^{T}+r_{32}(L-1) A_{c}^{T}(L-1) \Phi_{c}^{T}+r_{33}(L-1)\left(\Phi_{c}^{T}\right)^{L-1}\right) .
\end{aligned}
$$

If we subtract $r_{11}(L-2)$ from $r_{11}(L-1)$ and use (A-8), (A-14), (A-19) and (A-24), we obtain

$$
\begin{aligned}
& r_{11}(L-1)-r_{11}(L-2)=J_{1}(L-1, L-1) H B(L-1)+\sum_{i=1}^{L-2}\left(J_{1}(i, L-1)-J_{1}(i, L-2)\right) H B(i) \\
& =J_{1}(L-1, L-1) H B(L-1)-J_{1}(L-1, L-1) \sum_{i=1}^{L-2}\left(H \Phi^{(L-1)} J_{1}(i, L-2)+\Phi_{c}^{L} J_{2}(i, L-2)+\Phi_{c}^{(L-1)} J_{3}(i, L-2)\right) H B(i) \\
& =J_{1}(L-1, L-1)\left(H B(L-1)-\left(H \Phi^{(L-1)} r_{11}(L-2)+\Phi_{c}^{L} r_{21}(L-2)+\Phi_{c}^{(L-1)} r_{31}(L-2)\right)\right) .
\end{aligned}
$$

If we subtract $r_{12}(L-2)$ from $r_{12}(L-1)$ and use (A-8), (A-15), (A-20) and (A-25), we obtain

$$
\begin{aligned}
& r_{12}(L-1)-r_{12}(L-2)=J_{1}(L-1, L-1) \Phi_{c} B_{c}(L-1)+\sum_{i=1}^{L-2}\left(J_{1}(i, L-1)-J_{1}(i, L-2)\right) \Phi_{c} B_{c}(i) \\
& =J_{1}(L-1, L-1)\left(\Phi_{c} B_{c}(L-1)-\left(H \Phi^{(L-1)} r_{12}(L-2)+\Phi_{c}^{L} r_{22}(L-2)+\Phi_{c}^{(L-1)} r_{32}(L-2)\right)\right) .
\end{aligned}
$$

If we subtract $r_{13}(L-2)$ from $r_{13}(L-1)$ and use (A-8), (A-16), (A-21) and (A-26), we obtain

$$
\begin{aligned}
& r_{13}(L-1)-r_{13}(L-2)=J_{1}(L-1, L-1) B_{u v}(L-1)+\sum_{i=1}^{L-2}\left(J_{1}(i, L-1)-J_{1}(i, L-2)\right) B_{u v}(i) \\
& =J_{1}(L-1, L-1)\left(B_{u v}(L-1)-\left(H \Phi^{(L-1)} r_{13}(L-2)+\Phi_{c}^{L} r_{23}(L-2)+\Phi_{c}^{(L-1)} r_{33}(L-2)\right)\right) .
\end{aligned}
$$

If we subtract $r_{21}(L-2)$ from $r_{21}(L-1)$ and use (A-10), (A-14), (A-19) and (A-24), we obtain

$$
\begin{aligned}
& r_{21}(L-1)-r_{21}(L-2)=J_{2}(L-1, L-1) H B(L-1)+\sum_{i=1}^{L-2}\left(J_{2}(i, L-1)-J_{2}(i, L-2)\right) H B(i) \\
& =J_{2}(L-1, L-1)\left(H B(L-1)-\left(H \Phi^{(L-1)} r_{11}(L-2)+\Phi_{c}^{L} r_{21}(L-2)+\Phi_{c}^{(L-1)} r_{31}(L-2)\right)\right) .
\end{aligned}
$$

If we subtract $r_{22}(L-2)$ from $r_{22}(L-1)$ and use (A-10), (A-15), (A-20) and (A-25), we obtain

$$
\begin{aligned}
& r_{22}(L-1)-r_{22}(L-2)=J_{2}(L-1, L-1) \Phi_{c} B_{c}(L-1)+\sum_{i=1}^{L-2}\left(J_{2}(i, L-1)-J_{2}(i, L-2)\right) \Phi_{c} B_{c}(i) \\
& =J_{2}(L-1, L-1)\left(\Phi_{c} B_{c}(L-1)-\left(H \Phi^{(L-1)} r_{12}(L-2)+\Phi_{c}^{L} r_{22}(L-2)+\Phi_{c}^{(L-1)} r_{32}(L-2)\right)\right) .
\end{aligned}
$$

If we subtract $r_{23}(L-2)$ from $r_{23}(L-1)$ and use (A-10), (A-16), (A-21) and (A-26), we obtain

$$
\begin{aligned}
& r_{23}(L-1)-r_{23}(L-2)=J_{2}(L-1, L-1) B_{u v}(L-1)+\sum_{i=1}^{L-2}\left(J_{2}(i, L-1)-J_{2}(i, L-2)\right) B_{u v}(i) \\
& =J_{2}(L-1, L-1)\left(B_{u v}(L-1)-\left(H \Phi^{(L-1)} r_{13}(L-2)+\Phi_{c}^{L} r_{23}(L-2)+\Phi_{c}^{(L-1)} r_{33}(L-2)\right)\right) .
\end{aligned}
$$

If we subtract $r_{31}(L-2)$ from $r_{31}(L-1)$ and use (A-12), (A-14), (A-19) and (A-24), we obtain 


$$
\begin{aligned}
& r_{31}(L-1)-r_{31}(L-2)=J_{3}(L-1, L-1) H B(L-1)+\sum_{i=1}^{L-2}\left(J_{3}(i, L-1)-J_{3}(i, L-2)\right) H B(i) \\
& =J_{3}(L-1, L-1)\left(H B(L-1)-\left(H \Phi^{(L-1)} r_{11}(L-2)+\Phi_{c}^{L} r_{21}(L-2)+\Phi_{c}^{(L-1)} r_{31}(L-2)\right)\right) .
\end{aligned}
$$

If we subtract $r_{32}(L-2)$ from $r_{32}(L-1)$ and use (A-12), (A-15), (A-20) and (A-25), we obtain

$$
\begin{aligned}
& r_{32}(L-1)-r_{32}(L-2)=J_{3}(L-1, L-1) \Phi_{c} B_{c}(L-1)+\sum_{i=1}^{L-2}\left(J_{3}(i, L-1)-J_{3}(i, L-2)\right) \Phi_{c} B_{c}(i) \\
& =J_{3}(L-1, L-1)\left(\Phi_{c} B_{c}(L-1)-\left(H \Phi^{(L-1)} r_{12}(L-2)+\Phi_{c}^{L} r_{22}(L-2)+\Phi_{c}^{(L-1)} r_{32}(L-2)\right)\right) .
\end{aligned}
$$

If we subtract $r_{33}(L-2)$ from $r_{33}(L-1)$ and use (A-12), (A-16), (A-21) and (A-26), we obtain

$$
\begin{aligned}
& r_{33}(L-1)-r_{33}(L-2)=J_{3}(L-1, L-1) B_{u v}(L-1)+\sum_{i=1}^{L-2}\left(J_{3}(i, L-1)-J_{3}(i, L-2)\right) B_{u v}(i) \\
& =J_{3}(L-1, L-1)\left(B_{u v}(L-1)-\left(H \Phi^{(L-1)} r_{13}(L-2)+\Phi_{c}^{L} r_{23}(L-2)+\Phi_{c}^{(L-1)} r_{33}(L-2)\right)\right) .
\end{aligned}
$$

Here, the initial values of $r_{11}(k), r_{12}(k), r_{13}(k), r_{21}(k), r_{22}(k), r_{23}(k), r_{31}(k), r_{32}(k)$ and $r_{33}(k)$, at $k=0$ are $r_{11}(0)=0, r_{12}(0)=0, r_{13}(0)=0, r_{21}(0)=0, r_{22}(0)=0, r_{23}(0)=0, r_{31}(0)=0, r_{32}(0)=0$ and $r_{33}(0)=0$ from (A-14)-(A-16), (A-19)-(A-21) and (A-24)-(A-26).

Let us introduce the functions

$$
\begin{aligned}
& S_{11}(L)=\Phi^{L} r_{11}(L)\left(\Phi^{T}\right)^{L}, S_{12}(L)=\Phi^{L} r_{12}(L)\left(\Phi_{c}^{T}\right)^{L}, S_{13}(L)=\Phi^{L} r_{13}(L)\left(\Phi_{c}^{T}\right)^{L}, \\
& S_{21}(L)=\Phi_{c}^{L} r_{21}(L)\left(\Phi^{T}\right)^{L}, S_{22}(L)=\Phi_{c}^{L} r_{22}(L)\left(\Phi_{c}^{T}\right)^{L}, S_{23}(L)=\Phi_{c}^{L} r_{23}(L)\left(\Phi_{c}^{T}\right)^{L} \\
& S_{31}(L)=\Phi_{c}^{L} r_{31}(L)\left(\Phi^{T}\right)^{L}, S_{32}(L)=\Phi_{c}^{L} r_{32}(L)\left(\Phi_{c}^{T}\right)^{L}, S_{33}(L)=\Phi_{c}^{L} r_{33}(L)\left(\Phi_{c}^{T}\right)^{L} .
\end{aligned}
$$

Substituting (A-28)-(A-36) into (A-37) and introducing $G_{1}(L)=\Phi^{L} J_{1}(L, L), \quad G_{2}(L)=\Phi_{c}^{L} J_{2}(L, L)$ and $G_{3}(L)=$ $\Phi_{c}^{L} J_{3}(L, L)$, we obtain the recursive Equations (27)-(35) for $S_{11}(L), S_{12}(L), S_{13}(L), S_{21}(L), S_{22}(L), S_{23}(L)$, $S_{31}(L), S_{32}(L)$ and $S_{33}(L)$.

From (A-17), (A-22) and (A-27), $G_{1}(L), G_{2}(L)$ and $G_{3}(L)$ are formulated as

$$
\begin{aligned}
G_{1}(L) & =\left[K_{x}(L, L) H^{T}-\left(\Phi^{L} r_{11}(L) A^{T}(L) H^{T}+\Phi^{L} r_{12}(L) A_{c}^{T}(L) \Phi_{c}^{T}+\Phi^{L} r_{13}(L) A_{c}^{T}(L)\right)\right] R_{u}^{-1}(L) \\
& =\left[K_{x}(L, L) H^{T}-\left(S_{11}(L) H^{T}+S_{12}(L) \Phi_{c}^{T}+S_{13}(L)\right)\right] R_{u}^{-1}(L), \\
G_{2}(L) & =\left[K_{c}(L, L) \Phi_{c}^{T}-\left(\Phi_{c}^{L} r_{21}(L) A^{T}(L) H^{T}+\Phi_{c}^{L} r_{22}(L) A_{c}^{T}(L) \Phi_{c}^{T}+\Phi_{c}^{L} r_{23}(L) A_{c}^{T}(L)\right)\right] R_{u}^{-1}(L) \\
& =\left[K_{c}(L, L) \Phi_{c}^{T}-\left(S_{21}(L) H^{T}+S_{22}(L) \Phi_{c}^{T}+S_{23}(L)\right)\right] R_{u}^{-1}(L), \\
G_{3}(L) & =\left[R_{u}(L)-\left(\Phi_{c}^{L} r_{31}(L) A^{T}(L) H^{T}+\Phi_{c}^{L} r_{32}(L) A_{c}^{T}(L) \Phi_{c}^{T}+\Phi_{c}^{L} r_{33}(L) A_{c}^{T}(L)\right)\right] R_{u}^{-1}(L) \\
& =\left[R_{u}(L)-\left(S_{31}(L) H^{T}+S_{32}(L) \Phi_{c}^{T}+S_{33}(L)\right)\right] R_{u}^{-1}(L) .
\end{aligned}
$$

Substituting (27)-(29) into (A-38), after some manipulations, we obtain (36). Similarly, from (30)-(32) and (A-39), (37) is obtained. Also, by substituting (33)-(35) into (A-40), (38) is obtained.

Putting

$$
P_{1}(k, L)=\sum_{i=1}^{L} h(k, i, L) H K_{x}(i, i)\left(\Phi^{T}\right)^{-i},(\mathrm{~A}-41)
$$

$$
\begin{gathered}
P_{2}(k, L)=\sum_{i=1}^{L} h(k, i, L) \Phi_{c} K_{c}(i, i)\left(\Phi_{c}^{T}\right)^{-i}, \\
P_{3}(k, L)=\sum_{i=1}^{L} h(k, i, L) R_{u}(i)\left(\Phi_{c}^{T}\right)^{-i},
\end{gathered}
$$

from (3), (5) and (16), $h(k, L, L)$ satisfies 


$$
\begin{aligned}
& h(k, L, L) R_{u}(L)=B(k) A^{T}(L) H^{T}-\sum_{i=1}^{L} h(k, i, L)\left(H B(i) A^{T}(L) H^{T}+\Phi_{c} B_{c}(i) A_{c}^{T}(L) \Phi_{c}^{T}+R_{u}(i)\left(\Phi_{c}^{T}\right)^{L-i}\right) \\
& =K_{x}(k, k)\left(\Phi^{T}\right)^{L-k} H^{T}-\sum_{i=1}^{L} h(k, i, L)\left(H K_{x}(i, i)\left(\Phi^{T}\right)^{-i}\left(\Phi^{T}\right)^{L} H^{T}+\Phi_{c} K_{c}(i, i)\left(\Phi_{c}^{T}\right)^{-i}\left(\Phi_{c}^{T}\right)^{L} \Phi_{c}^{T}+R_{u}(i)\left(\Phi_{c}^{T}\right)^{L-i}\right)(\mathrm{A}-44) \\
& =K_{x}(k, k)\left(\Phi^{T}\right)^{L-k} H^{T}-\left(P_{1}(k, L)\left(\Phi^{T}\right)^{L} H^{T}+P_{2}(k, L)\left(\Phi_{c}^{T}\right)^{L+1}+P_{3}(k, L)\left(\Phi_{c}^{T}\right)^{L}\right) .
\end{aligned}
$$

Subtracting $P_{1}(k, L-1)$ from $P_{1}(k, L)$ and using (A-6), (A-14), (A-19) and (A-24), we obtain

$$
\begin{aligned}
& P_{1}(k, L)-P_{1}(k, L-1)=h(k, L, L) H K_{x}(L, L)\left(\Phi^{T}\right)^{-L}+\sum_{i=1}^{L-1}(h(k, i, L)-h(k, i, L-1)) H K_{x}(i, i)\left(\Phi^{T}\right)^{-i} \\
& =h(k, L, L) H K_{x}(L, L)\left(\Phi^{T}\right)^{-L}-h(k, L, L) \sum_{i=1}^{L-1}\left(H \Phi^{L} J_{1}(i, L-1)+\Phi_{c}^{L+1} J_{2}(i, L-1)+\Phi_{c}^{L} J_{3}(i, L-1)\right) H K_{x}(i, i)\left(\Phi^{T}\right)^{-i} \\
& =h(k, L, L)\left(H K_{x}(L, L)\left(\Phi^{T}\right)^{-L}-\left(H \Phi^{L} r_{11}(L-1)+\Phi_{c}^{L+1} r_{21}(L-1)+\Phi_{c}^{L} r_{31}(L-1)\right)\right) .
\end{aligned}
$$

Subtracting $P_{2}(k, L-1)$ from $P_{2}(k, L)$ and using (A-6), (A-15), (A-20) and (A-25), we obtain

$$
\begin{aligned}
& P_{2}(k, L)-P_{2}(k, L-1)=h(k, L, L) \Phi_{c} K_{c}(L, L)\left(\Phi_{c}^{T}\right)^{-L}+\sum_{i=1}^{L-1}(h(k, i, L)-h(k, i, L-1)) \Phi_{c} K_{c}(i, i)\left(\Phi_{c}^{T}\right)^{-i} \\
& =h(k, L, L)\left(\Phi_{c} K_{c}(L, L)\left(\Phi_{c}^{T}\right)^{-L}-\left(H \Phi^{L} r_{12}(L-1)+\Phi_{c}^{L+1} r_{22}(L-1)+\Phi_{c}^{L} r_{32}(L-1)\right)\right) .
\end{aligned}
$$

Subtracting $P_{3}(k, L-1)$ from $P_{3}(k, L)$ and using (A-6), (A-16), (A-21) and (A-26), we obtain

$$
\begin{aligned}
& P_{3}(k, L)-P_{3}(k, L-1)=h(k, L, L) R_{u}(L)\left(\Phi_{c}^{T}\right)^{-L}+\sum_{i=1}^{L-1}(h(k, i, L)-h(k, i, L-1)) R_{u}(i)\left(\Phi_{c}^{T}\right)^{-i} \\
& =h(k, L, L)\left(R_{u}(L)\left(\Phi_{c}^{T}\right)^{-L}-\left(H \Phi^{L} r_{13}(L-1)+\Phi_{c}^{L+1} r_{23}(L-1)+\Phi_{c}^{L} r_{33}(L-1)\right)\right) .
\end{aligned}
$$

Putting

$$
q_{1}(k, L)=P_{1}(k, L)\left(\Phi^{T}\right)^{L},
$$

and using (A-37), we obtain (20).

Putting

$$
q_{2}(k, L)=P_{2}(k, L)\left(\Phi_{c}^{T}\right)^{L},
$$

and using (A-37), we obtain (21).

Putting

$$
q_{3}(k, L)=P_{3}(k, L)\left(\Phi_{c}^{T}\right)^{L},
$$

and using (A-37), we obtain (22).

From (16) and (A-3), we obtain

$$
h(k, s, k)=\Phi^{k} J_{1}(s, k) .
$$

From (A-14), (A-37), (A-41) and (A-48), $q_{1}(k, k)$ is derived as

$$
\begin{aligned}
q_{1}(k, k) & =P_{1}(k, k)\left(\Phi^{T}\right)^{k} \\
& =\Phi^{k} \sum_{i=1}^{k} J_{1}(i, k) H K_{x}(i, i)\left(\Phi^{T}\right)^{-i}\left(\Phi^{T}\right)^{k} \\
& =\Phi^{k} r_{12}(k)\left(\Phi_{c}^{T}\right)^{k}=S_{11}(k) .
\end{aligned}
$$

From (A-15), (A-37), (A-42) and (A-49), $q_{2}(k, k)$ is derived as

$$
\begin{aligned}
q_{2}(k, k) & =P_{2}(k, k)\left(\Phi_{c}^{T}\right)^{k} \\
& =\Phi^{k} \sum_{i=1}^{k} J_{1}(i, k) \Phi_{c} K_{c}(i, i)\left(\Phi_{c}^{T}\right)^{-i}\left(\Phi_{c}^{T}\right)^{k} \\
& =\Phi^{k} r_{12}(k)\left(\Phi_{c}^{T}\right)^{k}=S_{12}(k) .
\end{aligned}
$$

Similarly, from (A-16), (A-37), (A-43) and (A-50), $q_{3}(k, k)$ is derived as

$$
\begin{aligned}
q_{3}(k, k) & =P_{3}(k, k)\left(\Phi_{c}^{T}\right)^{k} \\
& =\Phi^{k} \sum_{i=1}^{k} J_{1}(i, k) R_{u}(i)\left(\Phi_{c}^{T}\right)^{-i}\left(\Phi_{c}^{T}\right)^{k} \\
& =\Phi^{k} r_{13}(k)\left(\Phi_{c}^{T}\right)^{k}=S_{12}(k) .
\end{aligned}
$$

From (A-44), (A-48), (A-49) and (A-50), we obtain

$$
\begin{aligned}
& h(k, L, L) R_{u}(L)=K_{x}(k, k)\left(\Phi^{T}\right)^{L-k} H^{T} \\
& -\left(q_{1}(k, L) H^{T}+q_{2}(k, L) \Phi_{c}^{T}+q_{3}(k, L)\right) .
\end{aligned}
$$

Substituting (20), (21) and (22) into (A-55), we obtain 
(19) for the smoother gain $h(k, L, L)$.

From (8) and (A-51), the filtering estimate $\hat{x}(L, L)$ of $x(L)$ becomes

$$
\hat{x}(L, L)=\sum_{i=1}^{L} h(L, i, L) y(i)=\sum_{i=1}^{L} \Phi^{L} J_{1}(i, L) y(i) .
$$

Introducing

$$
O_{1}(L)=\sum_{i=1}^{L} J_{1}(i, L) y(i)
$$

we have

$$
\hat{x}(L, L)=\Phi^{L} O_{1}(L) .
$$

Subtracting $O_{1}(L-1)$ from $O_{1}(L)$ and using (A-8), we have

$$
\begin{aligned}
O_{1}(L)-O_{1}(L-1) & =J_{1}(L, L) y(L)+\sum_{i=1}^{L-1}\left(J_{1}(i, L)-J_{1}(i, L-1)\right) y(i) \\
& =J_{1}(L, L) y(L)-J_{1}(L, L) \sum_{i=1}^{L-1}\left(H \Phi^{L} J_{1}(i, L-1)+\Phi_{c}^{L+1} J_{2}(i, L-1)+\Phi_{c}^{L} J_{3}(i, L-1)\right) y(i) \\
& =J_{1}(L, L)\left(y(L)-\left(H \Phi \hat{x}(L-1, L-1)+\left(\Phi_{c}\right)^{2} \hat{v}_{2}(L-1)+\Phi_{c} \hat{v}_{3}(L-1)\right)\right) .
\end{aligned}
$$

Here, the functions $\hat{v}_{2}(L, L), \hat{v}_{3}(L, L), O_{2}(L)$ and $\mathrm{O}_{3}(L)$ are introduced as follows.

$$
\begin{gathered}
\hat{v}_{2}(L, L)=\Phi_{V}^{L} O_{2}(L), \hat{v}_{3}(L, L)=\Phi_{c}^{L} O_{3}(L) \\
O_{2}(L)=\sum_{i=1}^{L} J_{2}(i, L) y(i), \\
O_{3}(L)=\sum_{i=1}^{L} J_{3}(i, L) y(i) .
\end{gathered}
$$

From (A-58) and (A-59) with

$$
G_{1}(L)=\Phi^{L} J_{1}(L, L),
$$

we obtain (24) updating the filtering estimate $\hat{x}(L, L)$ from $\hat{x}(L-1, L-1)$. The initial condition of (24) for $\hat{x}(L, L)$ at $L=0$ is $\hat{x}(0,0)=0$ from (A-57) and (A-58).

Subtracting $\mathrm{O}_{2}(L-1)$ from $\mathrm{O}_{2}(L)$ and using (A-10), (A-57), (A-58), (A-60) and (A-61), we have

$$
\begin{aligned}
O_{2}(L)-O_{2}(L-1) & =J_{2}(L, L) y(L)+\sum_{i=1}^{L}\left(J_{2}(i, L)-J_{2}(i, L-1)\right) y(i) \\
& =J_{2}(L, L)\left(y(L)-\left(H \Phi^{L} O_{1}(L-1)+\Phi_{c}^{L+1} O_{2}(L-1)+\Phi_{c}^{L} O_{3}(L-1)\right)\right) \\
& =J_{2}(L, L)\left(y(L)-\left(H \Phi \hat{x}(L-1, L-1)+\left(\Phi_{c}\right)^{2} \hat{v}_{2}(L-1, L-1)+\Phi_{c} \hat{v}_{3}(L-1, L-1)\right)\right) .
\end{aligned}
$$

Subtracting $O_{3}(L-1)$ from $O_{3}(L)$ and using (A-12), (A-57), (A-58), (A-60) and (A-61), we have

$$
\begin{aligned}
O_{3}(L)-O_{3}(L-1) & =J_{3}(L, L) y(L)+\sum_{i=1}^{L}\left(J_{3}(i, L)-J_{3}(i, L-1)\right) y(i) \\
& =J_{3}(L, L)\left(y(L)-\left(H \Phi^{L} O_{1}(L-1)+\Phi_{c}^{L+1} O_{2}(L-1)+\Phi_{c}^{L} O_{3}(L-1)\right)\right) \\
& =J_{3}(L, L)\left(y(L)-\left(H \Phi \hat{x}(L-1, L-1)+\left(\Phi_{c}\right)^{2} \hat{v}_{2}(L-1, L-1)+\Phi_{c} \hat{v}_{3}(L-1, L-1)\right)\right) .
\end{aligned}
$$

From (A-60) and (A-62) with $G_{2}(L)=\Phi_{c}^{L} J_{2}(L, L)$, we obtain (25) for the filtering estimate $\hat{v}_{2}(L, L)$. Also, from (A-60) and (A-63) with $G_{3}(L)=\Phi_{c}^{L} J_{3}(L, L)$, we obtain (26) for the filtering estimate $\hat{v}_{3}(L, L)$.

From (8), (A-6), (A-57), (A-58), (A-60) and (A-61), the fixed-point smoothing estimate $\hat{x}(k, L)$ is updated as

$$
\begin{aligned}
& \hat{x}(k, L)-\hat{x}(k, L-1)=h(k, L, L) y(L)+\sum_{i=1}^{L-1}(h(k, i, L)-h(k, i, L-1)) y(i) \\
& =h(k, L, L)\left(y(L)-\left(H \Phi \hat{x}(L-1, L-1)+\left(\Phi_{c}\right)^{2} \hat{v}_{2}(L-1, L-1)+\Phi_{c} \hat{v}_{3}(L-1, L-1)\right)\right) \\
& =h(k, L, L) y(L)-h(k, L, L)\left(H \Phi^{L} O_{1}(L-1)+\Phi_{c}^{L+1} O_{2}(L-1)+\Phi_{c}^{L} O_{3}(L-1)\right) \\
& =h(k, L, L)\left(y(L)-\left(H \Phi \hat{x}(L-1, L-1)+\left(\Phi_{c}\right)^{2} \hat{v}_{2}(L-1, L-1)+\Phi_{c} \hat{v}_{3}(L-1, L-1)\right)\right) .
\end{aligned}
$$

